\title{
Doxorubicin-Loaded Carbon Dots Lipid-Coated Calcium Phosphate Nanoparticles for Visual Targeted Delivery and Therapy of Tumor
}

This article was published in the following Dove Press journal: International Journal of Nanomedicine

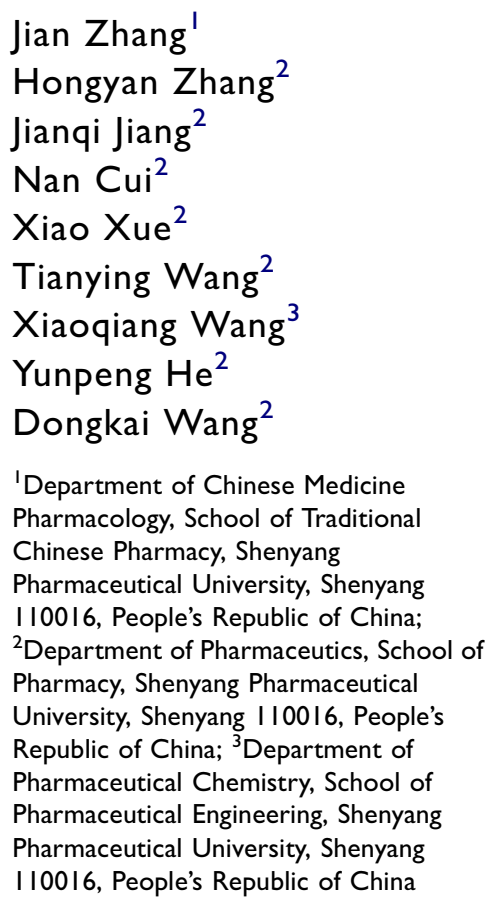

Correspondence: Dongkai Wang Department of Pharmaceutics, School of Pharmacy, Shenyang Pharmaceutical University, Shenyang II0016, People's Republic of China

Tel +86 24-43520529

Email wangycsyphu@I26.com
Background: Carbon dots (CDs) have attracted extensive attention in recent years because of their high biocompatibility and unique optical property. But they could not be well applied in the drug delivery system to enable distribution in tumor sites with their low $\mathrm{pH}$ sensitivity. They are barriers for drug delivery. CDs as an imaging proper were conjugated with doxorubicin (DOX) lipid-coated calcium phosphate (LCP) nanoparticle, for a $\mathrm{pH}$-sensitive nanocarrier and delivery of the antitumor drugs.

Materials and Methods: CDs were prepared by one-step hydrothermal treatment of citric acid and ethylenediamine. The nanoparticles were simply prepared by using microemulsion technology to form calcium phosphate $(\mathrm{CaP})$ core and further coated with cationic lipids.

Results: The structure was characterized by FTIR, XRD and TEM. In vitro release study revealed that DOX-CDs@LCP was pH dependent. The cytotoxicity assay demonstrated that it exhibited enhanced efficiency compared to the control group (DOX-CDs), but weaker than free DOX. The cellular uptake revealed that these $\mathrm{pH}$-sensitive nanoparticles could be taken up effectively and deliver DOX into the cytoplasm to reach antitumor effect. The fluorescence imaging indicated that DOX-CDs@LCP mostly distributed in the tumor region due to the enhanced permeability and retention effect (EPR) to reduce its systematical toxicity. Importantly, an antitumor activity study demonstrated that the DOX-CDs@LCP nanoparticles had higher antitumor activity than any other groups and lower toxicity. The results showed that $\mathrm{LCP}$ could significantly promote the release in tumor microenvironment due to $\mathrm{pH}$-response. The DOX-CDs could enhance load capacity and reduce drug premature releasing; real-time tracking of efficacy as confocal imaging contrast agent. Thus, DOX-CDs@LCP had antitumor capacity and lower systematic toxicity in tumor therapy.

Conclusion: DOX-CDs@LCP were proven as a promising tumor pH-sensitive and imaging-guided drug delivery system for liver cancer chemotherapy.

Keywords: carbon dots, calcium phosphate photodynamic therapy, $\mathrm{pH}$-sensitive, bioimaging, tumor targeting, therapy

\section{Introduction}

In the past decades, hepatic cancer is a major malignant tumor with high morbidity and fatality rate in the world. ${ }^{1,2}$ Although several approaches are being applied in clinical therapy, cancer is still a great damage to human life. There are some problems that should be noticed. 1) Many patients would already have reached the middle or terminal stages of cancer when diagnosed for the first time. Because the cancer was not diagnosed at the right time, they lose the best chance of surgery. ${ }^{3}$ 
2) Chemotherapy still plays an important role in the treatment of cancer. ${ }^{4,5}$ However, the effect of drugs on each patientvaries despite using the same chemotherapeutic drugs, thus requiring a real-time tracking of efficacy and timely adjustment of the dose to achieve the most effective therapy. ${ }^{6}$ 3) Severe side effects and systematic toxicity always limit the application of many antitumor drugs clinically. ${ }^{7}$ However, it has been reported that the extracellular $\mathrm{pH}$ of solid tumors is more acidic than normal tissues. ${ }^{8,9}$ Choosing a pH-sensitive drug delivery system is one of the ideal options for adding specific tissue distribution undergoing chemotherapy, as it could increase the therapeutical efficacy and reduce adverse effects. Therefore, it is necessary for researchers to find a multifunction carrier for efficient drug delivery to the tumor regions.

Optical imaging techniques have shown significant progress because of their great potential for applications in intelligent drug delivery systems. ${ }^{10-12}$ Carbon dots (CDs), as novel fluorescent materials, ${ }^{13}$ are also becoming promising drug carriers for disease treatment. ${ }^{14-16}$ Therefore, a suitable drug delivery system should be designed in whichthe dose of adverse effects can be controlled.

Doxorubicin (DOX), a model chemotherapeutic drug, is one of the most effective and highly regarded anticancer agents in cancer therapy due to its broad spectrum. However, despite being a chemotherapeutic drug, DOX is toxic to normal organs or cells as well, especially myocardial cells. ${ }^{17-19}$ These characters limit its clinical application. Therefore, CDs could combine with $\mathrm{DOX}^{16}$ and can noninvasively monitor the progression of drug and therapy in real-time adjustment. However, there is a contradictory limit in drugs delivered into tumors. When administered intravenously, the agents will tend to circulate for a long time if their size is not so small so that they could be excreted by the kidney, or if their size is large they could be rapidly recognized and trapped via the reticuloendothelial system (RES). ${ }^{14}$ In addition, it was reported that the $\mathrm{pH}$ of tumor tissues (6.$0-5.0$ ) is lower than that of normal tissues and in blood $(7.0-7.2){ }^{20,21}$ In order to decrease degradation in the lysosome and release drug in the cytoplasm, the calcium phosphate $(\mathrm{CaP})$ was identified as a preferable drug carrier. The nanomaterials $(\mathrm{CaP})$ that are approved for human use by $\mathrm{FDA}^{22}$ are extensively explored for drug/gene/vaccine delivery ${ }^{19,23,24}$ as well as for adequate biodegradation, excellent biocompatibility and high drug-loading efficacy. ${ }^{25,26}$ One of the advantages of $\mathrm{CaP}$ nanomaterials is the suitable size of particles to improve the distribution by EPR, because of good tumor penetration or retention depening on small or large particle size, respectively. ${ }^{27-30}$ Another is the $\mathrm{pH}$ sensitive release in the tumor microenvironment, which can increase the local concentration of chemotherapeutic drugs to enhance therapy in the acidic tumor microenvironment. ${ }^{25}$ Therefore, we hypothesized that DOX-loaded CD liposomes-Ca/P (DOX-CDs@LCP) would improve the drugloading efficiency, ${ }^{31}$ concentrate the distribution of drugs for minimal toxic side effects ${ }^{32,33}$ and monitor the response of chemotherapy laying a foundation for precise control. ${ }^{34,35}$

In this work, we synthesize a new agent of lipid-coated calcium phosphate nanoparticles (LCP NPs). It is made up of the inner $\mathrm{CaP}$ core and the outer cationic lipid shell. The LCP nanoparticles provide a nonfouling functionalizable matrix for drug loading, while CDs serve dual functions as both matrix for drug loading and imaging agent. Therefore, a drug delivery system (DOX-CDs@LCP NPs) was constructed. The LCP could be resolved in an acidic environment, and then release drug to the cytoplasm. As is shown in Scheme 1, DOX-CDs@LCP NPs could concentrate the tumor via EPR effect and resolve at lower $\mathrm{pH}$. Then, DOX-CDs could release the drugs from nanoparticles rapidly and reach the cytoplasm, and then accumulate in microtubules to exert their antitumor effects. DOX-CDs were monitored to adjust the treatment by loading capability and $\mathrm{pH}$-response release. The particle size distribution of DOX-CDs@LCP NPs was examined by Malvern Zetasizer, and the in vitro drug release, cell cytotoxicity and cellular uptake were measured. Finally, the in vivo antitumor activities were also investigated in the tumor-bearing mice. These nanoparticles exhibit superior abilities in bioimaging and intracellular drug delivery.

\section{Experimental}

\section{Chemicals and Reagents}

Citric acid, PEG-2000, mPEG2000-DSPE, DOPA (1,2-dioleoyl-sn-glycerol-3-phosphate) and DOPC (1,2-dioleoyl-snglycero-3-phosphocholine) were purchased from Sinopharm Chemical Reagent Co., Ltd. (Shanghai, China). Doxorubicin hydrochloride (Igepal ${ }^{\circledR}$ CO-520) was obtained from SigmaAldrich Chemical Co (St. Louis, MO, USA). Cholesterol was purchased from Shanghai Advanced Vehicle Technology Ltd Co (Shanghai). Dichloromethane (DCM) and dimethyl sulfoxide (DMSO) were supplied by Yuwang Chemical Reagent Factory (Shandong, China). Ethylenediamine anhydrous was purchased from Damao Chemical Reagent Factory (Tianjin, 


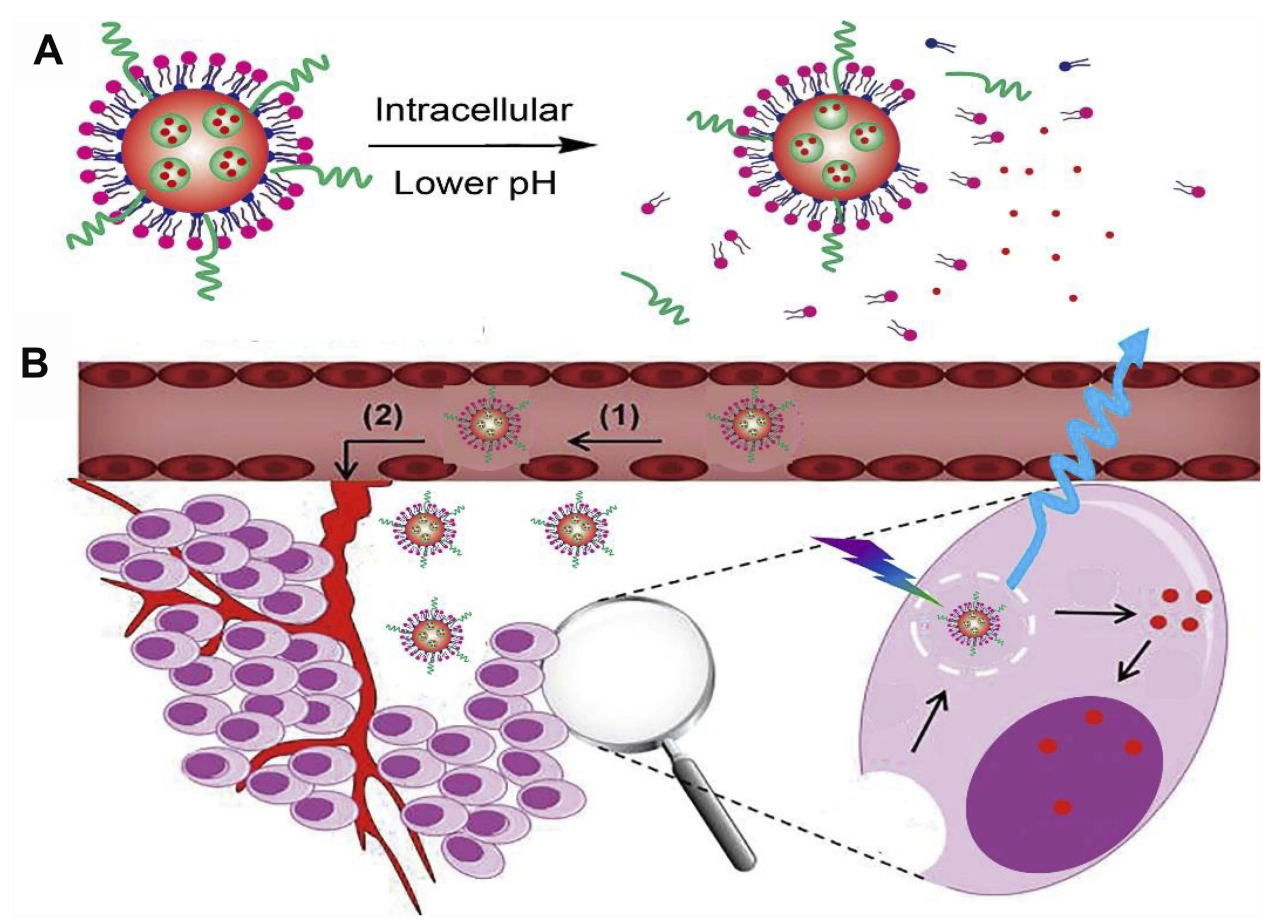

Scheme I The DOX-CDs@LCP accumulated at the tumor site by the EPR effect $(\mathbf{B})$ and CDs-DOX released at the low $\mathrm{pH}$ sites $(\mathbf{A})$.

China). 3-(4,5-dimethylthialzol-2-yl) -2,5-diphenyltetrazolium bromide (MTT), Dulbecco's modified Eagle's medium (DMEM), phosphate-buffered saline (PBS), fetal bovine serum (FBS) and 4', 6-diamidino-2-phenylindole (DAPI) were supplied by Sigma-Aldrich.

\section{Synthesis of DOX-CDs}

CDs were prepared by the hydrothermal method. ${ }^{15}$ First, citric acid $(2.5 \mathrm{~g})$ was dissolved in demonized water $(20 \mathrm{~mL})$, and ethylenediamine $(5 \mathrm{~mL})$ was injected at room temperature. And then, the solution was transferred into a stainless steel autoclave and heated at $160^{\circ} \mathrm{C}$ for $8 \mathrm{hrs}$. Further, the crude product was purified by a dialysis bag $(\mathrm{Mw}=3500)$ for $24 \mathrm{hrs}$. The final solution was lyophilized and dry CDs were obtained. The fluorescence quantum yield of the CDs was calculated as given in the previous report.

\section{Preparation of DOX@LCP and DOX-CDs@LCP}

DOPA-coated DOX-CDs NPs (DOX-CDs@DOPA NPs) were prepared by microreactor method. ${ }^{28}$ Briefly, $150 \mu \mathrm{L}$ of $\mathrm{CaCl}_{2}(500 \mathrm{mM})$ with $\mathrm{CDs}(10 \mathrm{mg} / \mathrm{mL})$ and $100 \mu \mathrm{L}$ of DOX $(5 \mathrm{mg} / \mathrm{mL})$ were dispersed in the cyclohexane oil phase ( $8 \mathrm{~mL}$ ) (cyclohexane:Igepal CO-520=79:21,v/v) to form a well-dispersed water-in-oil microemulsion. Then, the phosphate microemulsion was dispersed by $150 \mu \mathrm{L}$ of $\mathrm{Na}_{2}$
$\mathrm{HPO}_{4}(50 \mathrm{mmol} / \mathrm{L})$ in a separate oil phase. And $100 \mu \mathrm{L}$ of DOPA $(20 \mathrm{mM})$ in chloroform was added in the phosphate solution. After mixing both the microemulsions for half an hour, the two microemulsions were combined, then the microemulsion was stirred for an additional $0.5 \mathrm{hrs}$ to yield DOX-CDs@DOPA NPs. Then, 16 mL of absolute ethanol was added in the microemulsion, and the mixtures were centrifuged at $12,000 \mathrm{~g}$ for $0.25 \mathrm{hrs}$ to collect DOX-CDs @DOPA NPs. The resultant DOX-CDs@DOPA NPs were washed two times with ethanol and once with ethanol to remove excess DOPA, then it was dried with nitrogen atmosphere. The DOX-CDs@DOPA NPs were dissolved in chloroform and stored in a glass vial at $-20^{\circ} \mathrm{C}$ for use.

To prepare the final DOX-CDs@LCP NPs, some materials were compounded previously. In brief, DOPC, cholesterol, DSPE-PEG-2000 and DSPE-PEG-biotin (weight ratio of 10:10:3:3) were mixed in $500 \mu \mathrm{L}$ of DOX-CDs@DOPA NPs. After evaporating the chloroform, the residual lipid was hydrated to form LCP NPs in $2.0 \mathrm{~mL}$ of $\mathrm{H}_{2} \mathrm{O}$.

In addition, DOX@LCP NPs were also prepared using the same method without CDs used.

\section{Characterization}

The particle sizes and zeta potential of the nanoparticles were measured by Malvern Zetasizer Nano ZS90 (Malvern, UK) of different formulations. The morphology of the 
nanoparticles was characterized by TEM (JEM-1400, JEOL Ltd, Tokyo, Japan). And the DOX-CDs@LCP NPs were stained with a little of $2 \%$ phosphotungstic acid for 15 mins. Fourier transform infrared spectroscopy (FTIR) (VERTEX-70, Bruker, Germany) analysis was performed to identify the typical vibration properties of nanoparticles. The crystal structure was investigated by X-ray power diffraction (PXRD) (Bruker, D2 Phaser, Germany). Transmission electron micrograph (TEM) was observed by a microscope operated at $200 \mathrm{kV}$ (Tecnai G2 F30, FEI). A UV/vis spectrophotometer (UV-1700, SHIMADZU) was employed to obtain the UV/vis spectra. The fluorescence spectra were recorded on a multimode reader (VarioskanFlash 3001, Thermo Fisher Scientific Inc., Waltham, MA, US).

\section{Drug Loading and Encapsulation Efficiency}

To determine the drug-loading capacity of DOX, $1 \mathrm{mg}$ of DOX-CDs@LCP NPs and DOX@LCP NPs was stirred in $1 \mathrm{~mL}$ of $1 \mathrm{~N} \mathrm{HCl}$ solution for $0.5 \mathrm{hrs}$ to dissolve the shell of CaP. Subsequently, the solution was centrifuged at $12,000 \mathrm{rpm}$ for about $10 \mathrm{mins}$. The concentration of DOX in nanoparticles of supernatant was used to estimate by UV-Vis spectrometer at $480 \mathrm{~nm}$. A calibration curve of DOX was prepared following the same previous condition to calculate the amount of DOX encapsulated in the DOXCDs@LCP NPs. The drug-loading capacity (DLC) was defined as the following equation:

$$
\operatorname{DLC}(\%)=\frac{\text { weight of DOX }}{\text { total weight of complexes }} \times 100
$$

\section{In vitro $\mathrm{pH}$-Sensitive Drug Release}

The pH-dependent release of DOX from DOX-CDs@LCP NPs and DOX@LCP NPs was studied by a dialysis method in a different buffer ( $\mathrm{pH} 7.4$ and 5.5) at $37^{\circ} \mathrm{C}$, respectively. In brief, DOX-CDs@LCP NPs (5 mg) and DOX@LCP NPs $(5 \mathrm{mg})$ were dispersed in PBS $(5 \mathrm{~mL})$ for the solutions $(1 \mathrm{mg} /$ $\mathrm{mL}$ ) and then were sealed in a dialysis bag. Subsequently, the dialysis bag (the trapped molecular weight is $3.5 \mathrm{kDa}$ ) was immersed in $50 \mathrm{~mL}$ of PBS at $37^{\circ} \mathrm{C}$ with continuous shaking. The flask was placed in a shaking incubator (stirring speed: $100 \mathrm{rpm})$ at $37^{\circ} \mathrm{C}$. At specific time intervals $(0,1,2,4,8,12$, $24,36 \mathrm{hrs})$, the solution ( $5 \mathrm{~mL}$ ) outside the dialysis bag was taken and replaced with the same volume of fresh PBS. The concentration of released DOX in the release medium was quantified and analyzed using a UV-vis spectrophotometer at $480 \mathrm{~nm}$.

\section{Cytotoxicity Assay}

Cell Culture

The human hepatocellular carcinoma cell line (HepG2 cells) was donated by the Wu's Pharmacology Laboratory of Shenyang Pharmaceutical University (Shengyang, China) and grown in RPMI-1640 medium (Gibco, Grand Island, NY, USA) supplemented with 10\% FBS (Gibco), 100 $\mathrm{IU} \mathrm{mL} \mathrm{m}^{-1}$ penicillin and $100 \mathrm{mg} / \mathrm{mL}$ streptomycin at $37^{\circ} \mathrm{C}$ in a humidified incubator with $5 \% \mathrm{CO}_{2}$ atmosphere. Cells in the exponential phase of growth were used in the experiments.

\section{Cellular Uptake Studies of DOX-CDs@LCP NPs}

The cytotoxicity of DOX-CDs@LCP NPs against HepG2 cells was determined by MTT assay. In brief, HepG2 cells were seeded in a 96-well plate at a density of $1 \times 10^{4}$ cells per well and incubated overnight at $37^{\circ} \mathrm{C}$ and $5 \% \mathrm{CO}_{2}$. Then, the fresh medium containing free DOX or DOXCDs@LCP NPs was added to each well after the culture medium was removed. After 24 and $48 \mathrm{hrs}, 20 \mu \mathrm{L}$ of MTT solution $(5 \mathrm{mg} / \mathrm{mL})$ was added in each well and further incubated for $4 \mathrm{hrs}$. Afterward, the solution was measured at $570 \mathrm{~nm}$ by a SpectraMax $^{\circledR}$ M3 microplate reader (Molecular Devices Corporation, CA, USA) by MTT method. Cell viability was calculated using the following formula:

$$
\text { Cell viability }(\%)=\frac{\mathrm{A}_{\text {(test group) }}}{\mathrm{A}_{(\text {control group })}} \times 100
$$

\section{In vitro Intracellular Uptake}

The cellular uptake of free DOX and DOX-CDs@LCP NPs was determined by flow cytometry. A549 cells were grown overnight at a density of $3 \times 10^{5}$ cells per well in a 6-well plate. Subsequently, cells were exposed to free DOX or DOX-CDs@LCP NP complexes for different times (1, 2 and $4 \mathrm{hrs})$; after that, the cells were washed twice with phosphate-buffered saline and centrifuged at $1000 \mathrm{rpm}$ for 5 mins to collect cells. Finally, cells were resuspended in PBS for three times, and the uptake of DOX was analyzed using a FACS Calibur flow cytometer (BD Biosciences, CA, USA).

\section{Confocal Microscopy}

HepG-2 cells were seeded in a 6-well plate at a cell density of $1 \times 10^{5}$ cell/well and cultured for $24 \mathrm{hrs}$. After incubation, the medium was replaced by the medium containing DOX, DOX-CDs and DOX-CDs@LCP (equal amount of DOX, $5 \mu \mathrm{g} / \mathrm{mL}$ ) for $4 \mathrm{hrs}$, respectively. The 
cells were washed three times with PBS and fixed with 4\% paraformaldehyde at $25^{\circ} \mathrm{C}$ for 15 mins. To stain and visualize nuclei, DAPI was added afterward. Last, the cells were washed three times with cold PBS and sealed on the glass slide, which were observed with a confocal laser scanning microscopy (CLSM, Model $\mathrm{C}_{2}^{+}$Nikon Ltd., Tokyo, Japan).

\section{In vivo Biodistribution Assay}

In the biodistribution assay, drugs can be chosen as the fluorescence probe to image the site of cancer and investigate the cancer-targeting efficacy of mice. The tumorbearing mice were prepared by the subcutaneous injection of $\mathrm{H} 22$ cells to Kunming mice, and the subcutaneous H22 tumor-bearing mice were intravenously injected with DOX, DOX-CDs and DOX-CDs@LCP nanoparticle solution $(200 \mu \mathrm{L}, 0.2 \mathrm{mg} / \mathrm{mL})$. After 1, 2, 4, 8, 12 and $24 \mathrm{hrs,}$ the mice were bioimaged using a luminescent image analyzer (Bruker, CA) to investigate their body distribution.

\section{Animal and Tumor Model}

The animals named Kunming (KM) mice (male, 6 weeks) were obtained from Shenyang Pharmaceutical University Experimental Animal Center (Shenyang, China). All the animal studies were carried on in agreement with the guidelines of the Experimental Animal Administrative Center and ratified by the Animal Ethical Committee of Shenyang Pharmaceutical University. The antitumor activity in vivo was assessed in KM mice implanted sarcoma using $\mathrm{H} 22$ cells. The $\mathrm{H} 22$ cells $\left(2 \times 10^{7}\right.$ cells $/ \mathrm{mL}$, suspended in $200 \mu \mathrm{L}$ PBS) were injected subcutaneously into the right anterior armpit of $\mathrm{KM}$ mice. When the tumor reached $\sim 100 \mathrm{~mm}^{3}$, they were divided randomly into several groups (control, free DOX, DOX-CDs, DOXCDs@LCP nanoparticles, n=10) and marked the number using picric acid.

\section{In vivo Antitumor Evaluation}

The in vivo antitumor effect of nanoparticles was investigated using an H22 tumor xenograft model on Kunming mice. In brief, $\mathrm{H} 22$ cells were obtained and diluted to $1 \times 10^{7}$ cells $/ \mathrm{mL}$ with normal saline. Then, H22 cells $(0.2 \mathrm{~mL})$ were carefully injected subcutaneously into the right limb armpit of the mice. When tumors were $\sim 100 \mathrm{~mm}^{3}$ in size, the tumor-bearing mice were randomly separated into four groups (10 mice each group) and were injected intravenously via the tail vein with normal saline, free DOX, DOXCDs and DOX-CDs @LCP nanoparticles, respectively. The concentration of drugs is an equal dose of DOX $5 \mathrm{mg} / \mathrm{kg}$ body weight. Tumor volume was calculated using the following equation:

$$
\text { Tumor volume }=\frac{\mathrm{L}-\mathrm{W}^{2}}{2}
$$

where $L$ means the tumor length and $W$ means the tumor width.

Tumor volume and body weight of each mice were measured every 2 days for 19 days. The tumor size was determined with a caliper in two dimensions. On the 19th day, mice were sacrificed and the tumor tissues were harvested to measure the tumor weight. The antitumor activity was evaluated in terms of tumor weight (g). The tumor growth inhibition rate (IR) was calculated using the following equation:

$$
\mathrm{IR}(\%)=\frac{\mathrm{W}_{1}-\mathrm{W}_{2}}{\mathrm{~W}_{1}} \times 100
$$

where $\mathrm{W}_{1}$ represents the tumor weight of the control group with saline and $\mathrm{W}_{2}$ represents the treatment group with drug.

\section{Statistical Analysis}

All results were indicated as mean $\pm \mathrm{SD}$ and tested at least three times. The data were analyzed by the analysis of variance (ANOVA) among at least three groups and a Student's $t$-test between two groups by using SPSS 13.0 software (SPSS Inc., Chicago, IL, USA). Student's $t$-test P-values $0.05,0.01$ were considered statistically significant differences.

\section{Results and Discussion Synthesis and Characterization of DOX-CDs@LCP and DOX-CDs}

In this study, we constructed a multifunctional $\mathrm{pH}$-sensitive and imageable drug delivery system for the chemotherapeutic agent DOX. First, CDs with high quantum yield were synthesized following a published method. ${ }^{12}$ The synthetic routes of CDs are shown in Scheme 1. The average particle diameters of CDs and DOX-CDs@LCP NPs were 4.02 and $97.3 \mathrm{~nm}$ by dynamic light scattering (DLS) measurement, respectively (Figure 1A). The polydispersity indexes (PDI) of CDs and DOX-CDs@LCP NPs were 0.075 and 0.212, respectively, indicating uniform particle size and narrow distribution of these nanoparticles.

The FTIR was used to investigate the chemical groups on the surface of the materials and analyze the interaction among drugs, CDs and LCP. The FTIR patterns of DOX- 


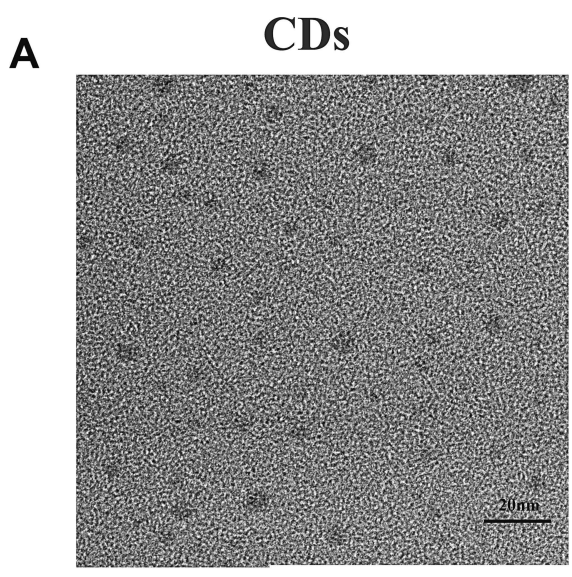

B

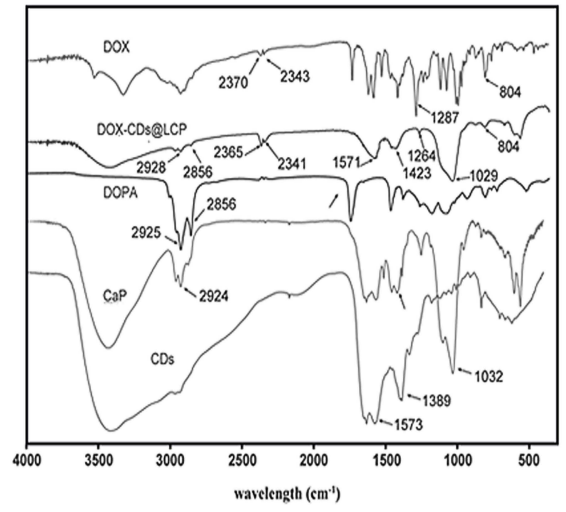

DOX-CDs@LCP

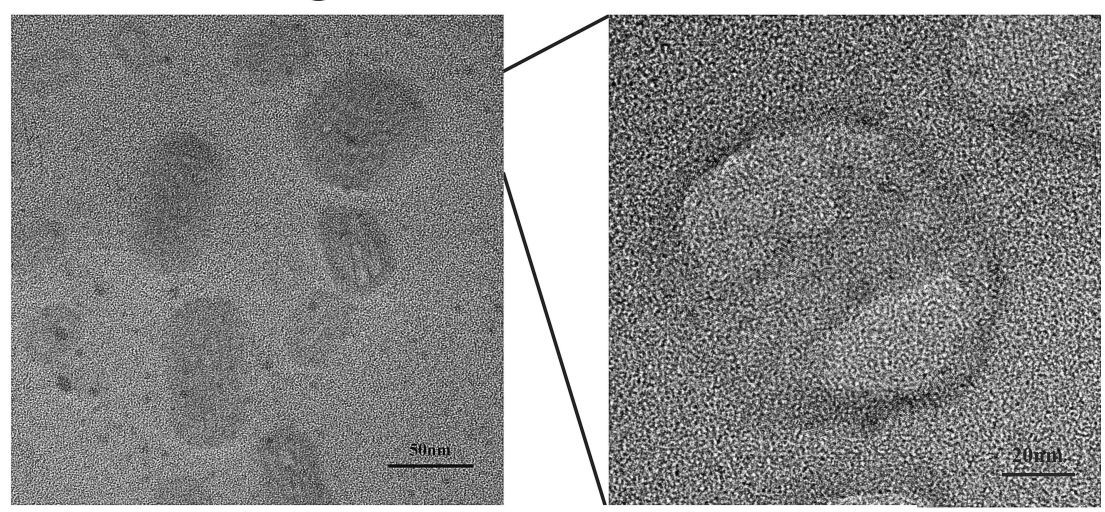

C

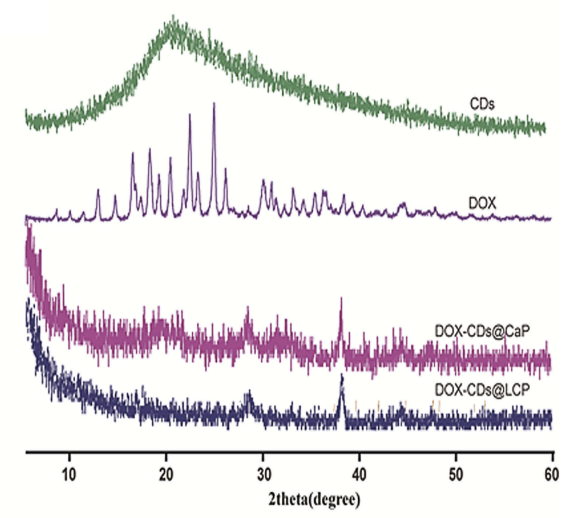

Figure I TEM image of CDs and DOX-CDs@LCP (A), and the spectra $(\mathbf{B})$ and X-ray diffractograms $(\mathbf{C})$ of CDs, DOX, DOX-CDs@CaP and DOX-CDs@LCP.

CDs@LCP and materials were coincident, which indicated that the drugs and LCP did not interact with each other.

The FTIR spectra of CDs, DOX-CDs@LCP, DOPA, CaP and CDs are shown in Figure 1B. The broad band at $3415 \mathrm{~cm}^{-1}$ was assigned to the stretching vibrations of $\mathrm{NH}_{2}$ and $\mathrm{OH}$ groups. The $\mathrm{CH}$ stretching band is indicated by the peaks at $2926 \mathrm{~cm}^{-1}$. Compared to CDs, the band at $1631 \mathrm{~cm}^{-1}$ arises from the $-\mathrm{N}-\mathrm{H}$ deformation mode of $-\mathrm{N}-\mathrm{H}$ bending. The appearance of band at $1571 \mathrm{~cm}^{-1}$ is attributed to the $-\mathrm{OH}$ bending of carboxylic acid, whereas the band at $1385 \mathrm{~cm}^{-1}$ corresponds to - $\mathrm{C}-\mathrm{N}$ bond stretching of primary amine. The $\mathrm{C}-\mathrm{O}$ stretching of the carboxylic group is attributed by the peak at $1178 \mathrm{~cm}^{-1}$. The characteristic absorption peak at $1571 \mathrm{~cm}^{-1}$ of DOX-CDs@LCP was attributed to the backbone vibration of aromatic rings of DOX. The multiple absorption peaks between $1598 \mathrm{~cm}^{-1}$ and $1571 \mathrm{~cm}^{-1}$ of the complexes were the features of benzene ring of DOX, and FTIR (Figure 1B) confirmed the successful preparation of DOX-CDs@LCP complexes.

The PXRD patterns were measured to confirm the change in drug crystallinity. The PXRD patterns of DOX, CDs,
DOX-CDs@CaP and DOX-CDs@LCP are shown in Figure 1C. The DOX displayed many diffraction peaks, suggesting the strong crystallized structure of DOX. However, compared with the PXRD spectrum of DOX, the crystalline diffraction pattern was markedly weakened in DOX-CDs@CaP and DOX-CDs@LCP compared to pure DOX, suggesting the lowest degree of crystallinity.

\section{In vitro Drug Release}

The effect of drug release behavior from nanoparticles was measured in different $\mathrm{pH}$ buffer conditions by a dialysis bag. As is shown in Figure 2A, the nanoparticles in $\mathrm{pH} 5.5$ showed a burst release pattern of DOX and maintain

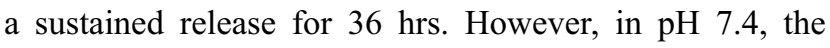
release rate of DOX from nanoparticles was significantly lower than the other. At $\mathrm{pH} 7.4$, as shown in Figure 2B, the release of DOX was notably inhibited, and about $40 \%$ of DOX was released. ${ }^{36}$ Then, it could be attributed to the impact of $\mathrm{CaP}$ in the outer shell of the nanoparticles, which might act as a diffusion barrier at $\mathrm{pH}$ 7.4. However, at a lower $\mathrm{pH}$, the release pattern of DOX was higher. Then, 
it indicates that most of the $\mathrm{CaP}$ on the outer shell are dissolved into an acidic environment and therefore no longer act as a diffusion barrier. Therefore, the release of DOX from DOX-CDs@LCP nanoparticles increased at lower $\mathrm{pH}$, so $\mathrm{pH}$-dependent agents of $\mathrm{CaP}$ greatly affected the release behavior of DOX in different $\mathrm{pH}$.

\section{Cytotoxicity Assay}

To explore the cell inhibitory effect and the $\mathrm{pH}$-sensitivity of DOX-CDs@LCP, the cytotoxicity of the DOX, DOX-CDs @LCP and CDs@LCP against HepG-2 cells was tested using the MTT assay (Figure 3). CDs@LCP as the free nanoparticlescould remove the impact of free nanoparticles. Then, the cytotoxicity of the DOX-CDs@LCP group was lower than the group of DOX at low concentrations $(0.01-0.1) \mu \mathrm{g} / \mathrm{mL}$, which may be attributed to the slow release of DOX from the complexes. Additionally, cell viability was inhibited apparently at high concentrations of DOX-CDs@LCP and DOX $(0.5-10 \mu \mathrm{g} / \mathrm{mL})$, which may be due to the increasing accumulation of DOX. Notably, DOX-CDs@LCP exhibited much lower tumor cytotoxicity than DOX, which could be ascribed to the different cellular uptake mechanism of DOX-CDs @ LCP and DOX. Free DOX enters the cell by passive diffusion, inducing high drug concentrations and causing cytotoxicity. Then tumor cells can be rapidly inhibited. By contrast, after the nanoparticles were internalized by tumor cells, DOX-CDs@LCPs enter the cells through endocytosis, which were released rather slowly, thus lowering its tumor cytotoxicity.

\section{Intracellular Uptake}

Flow cytometry was used for the quantification of intracellular uptake of control, DOX and DOX-CDs@LCP nanoparticles. It is shown in Figure 4 that the cellular uptake of nanoparticles increased with extension of incubation time; nevertheless, the uptake of DOX was slightly higher than DOX-CDs@LCP nanoparticles when HepG-2 cells were incubated for $2 \mathrm{hrs}$. The slight increase could be described that DOX was easier than DOX-CDs@LCP when the cells were incubated first $2 \mathrm{hrs}$. On the contrary, the uptake of DOX-CDs@LCP nanoparticles was higher than DOX when HepG-2 cells were incubated for $4 \mathrm{hrs,} \mathrm{which} \mathrm{was} \mathrm{more}$ significantly increased than before. Maybe it was fully absorbed by the cells, leading to the gradual binding of the nanoparticles with the fluorescence intensity of free DOX, which was in accordance with reported literature. ${ }^{31}$ It is reported that the uptake of CDs was higher than DOX since the intracellular uptake of DOX was through passive diffusion and CDs should be transported into cells through both endocytosis and passive diffusion. The difference may be ascribed to the fact that the $\mathrm{CaP}$ on the surface of $\mathrm{CDs}$ lowered their cellular uptake. ${ }^{32}$ However, it was still gratifying that the cellular uptake of the complexes reached at a high level when the cells were incubated for $4 \mathrm{hrs}$. Finally, this result indicated that DOX-CDs@LCP nanoparticles could be effectively absorbed by HepG2 cells.

The intracellular uptake of DOX and DOX-CDs@LCP was visualized by confocal laser scanning microscopy and flow cytometry. The fluorescence of the DOX and the complexes in the HepG-2 cells is shown in Figure 5. HepG-2 cells were incubated with DOX (control group), DOX-CDs and LCP, respectively, for 4 hrs. DAPI was employed for nuclear staining to localize where the drug distributed in the cell. As it was shown, the free DOX was distributed in the nucleus, the CDs-DOX was mainly distributed in the cytoplasm and the DOX-CDs@LCP was observed in the cytoplasm and nucleus. This may be ascribed to the fact that DOX-CDs@LCP released far more than CDs-DOX, then the released DOX penetrated into the nucleus, which was also consistent with in vitro release results. Whereas, this phenomenon would
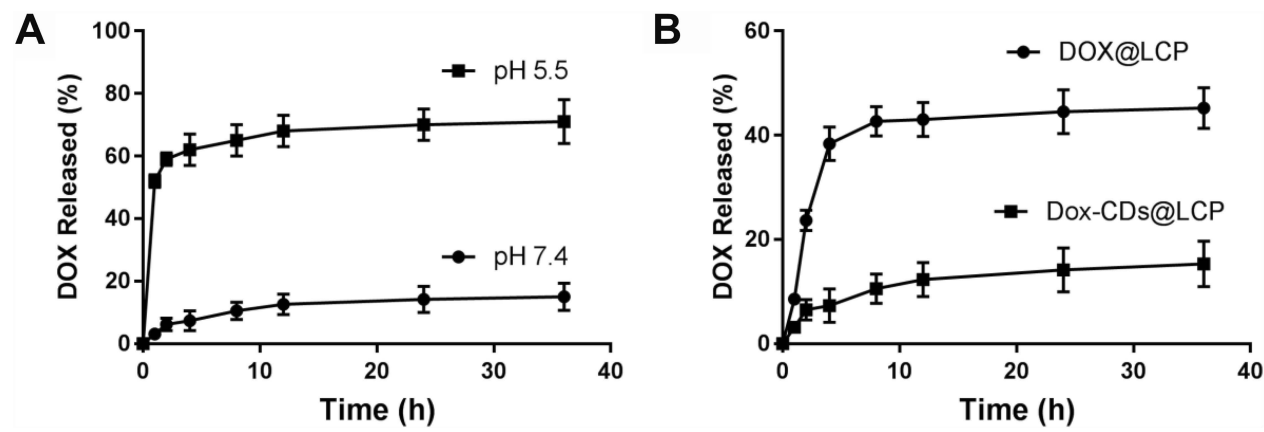

Figure 2 (A) In vitro release profile of DOX from DOX-CDs@LCP nanoparticles at $\mathrm{pH} 7.4$ and 5.5, n=3, respectively. (B) In vitro release profile of DOX from DOX-CDs @LCP nanoparticles and DOX@LCP nanoparticles at $\mathrm{pH} 7.4$, respectively, n=3. 


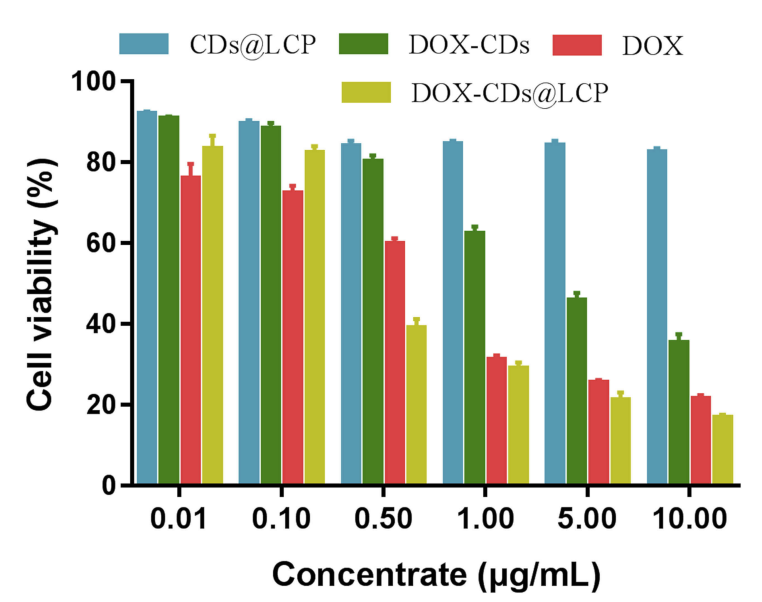

Figure 3 Cell viability of free DOX, DOX-CDs and CDs@LCP nanoparticles against HepG2 cells at different concentrations for $48 \mathrm{hrs}$.

assure effective intracellular delivery and lead to an advanced DOX concentration in the tumor site; it resulted in a higher level of cytotoxicity of the guided cells. Then, the enhanced amount of DOX release from nanoparticles might improve the therapeutic capacity in tumor cells.

\section{In vivo Real-Time Imaging of Biodistribution}

The in vivo biodistribution of nanoparticles in H22 tumorbearing mice was investigated using a non-invasive nearinfrared optical imaging technique. The tumor-bearing mice were injected with DOX, DOX-CDs and DOX-CDs@LCP nanoparticles. Figure 6 displays the real-time images of the tumor-bearing mice. During the living imaging test, most of the nanoparticles were visualized little in tumor at $4 \mathrm{hrs}$. However, preferential accumulation of fluorescence was

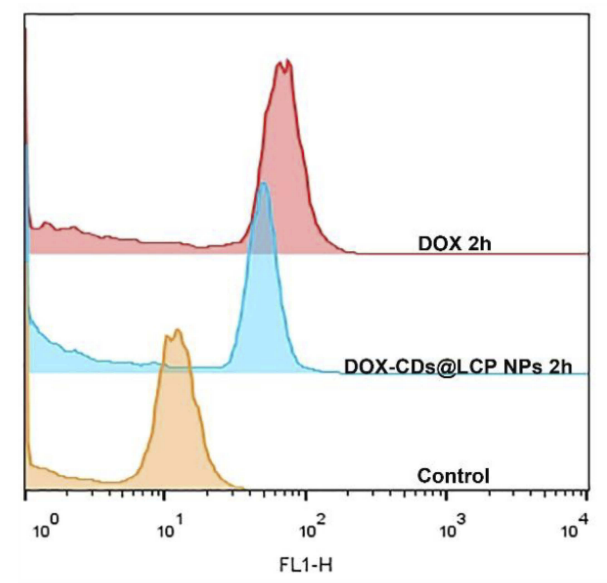

obvious in the tumor site other than other normal tissues at $24 \mathrm{hrs}$ after injection, which provided decisive evidence that the designed DOX-CDs@LCP were available for tumorspecific drug delivery with others. The tumor target of nanoparticles might be due to a combination of an enhanced permeability and retention (EPR) effect and receptor-mediated uptake of nanoparticles. Overall, these results indicate that DOX-CDs@LCP nanoparticles can be expected to be a highly efficient drug delivery vehicle to achieve targeted intracellular delivery of anticancer drugs.

\section{In vivo Antitumor Activities}

The in vitro results indicated DOX-CDs@LCP were satisfied and effective in antitumor study. Then, we started to perform a therapeutic efficacy study to assess the potential of different preparations for inhibiting tumor growth in vivo. The antitumor effect of nanoparticles is shown in Figure 7; compared with Con group, CDs DOX, CDs-DOX and DOXCDs@LCP group showed significant $(\mathrm{p}<0.01)$ in vivo antitumor activity, and the inhibition rates were $26.4 \%$, $46.1 \%$ and $53.6 \%$, respectively; however, the tumor growth of CDs group was higher than Con, demonstrating that this could not inhibit the tumor growth by the appropriate way of chemotherapy therapy and that the CDs nanoparticles may increase tumor growth.

The record of average tumor volumes and changes in body weight could illustrate the antitumor efficiency and toxicity during the experiment, respectively. As shown in Figure 7A, all groups except for DOX-CDs@LCP exhibited no significant difference in tumor volume in the first 5 days, while treatment groups inhibited the tumor growth to different degrees after 5 days. As expected, the control

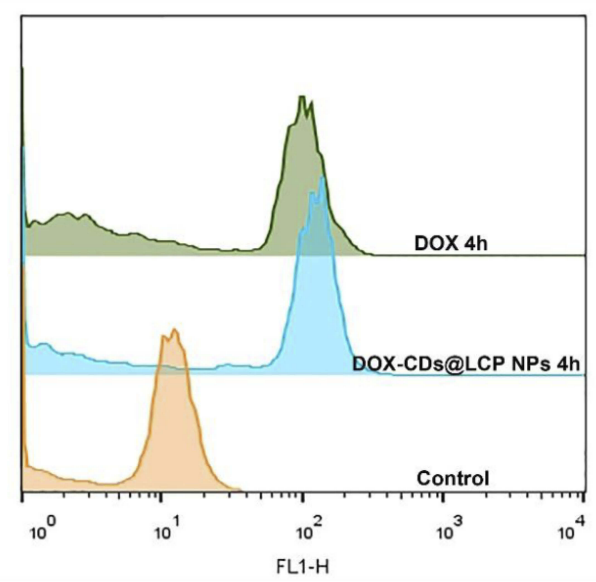

Figure 4 FCM results of cellular uptake in HepG2 cells after incubation with free DOX, DOX-CDs@LCP nanoparticles incubated for 2 and 4 hrs. 


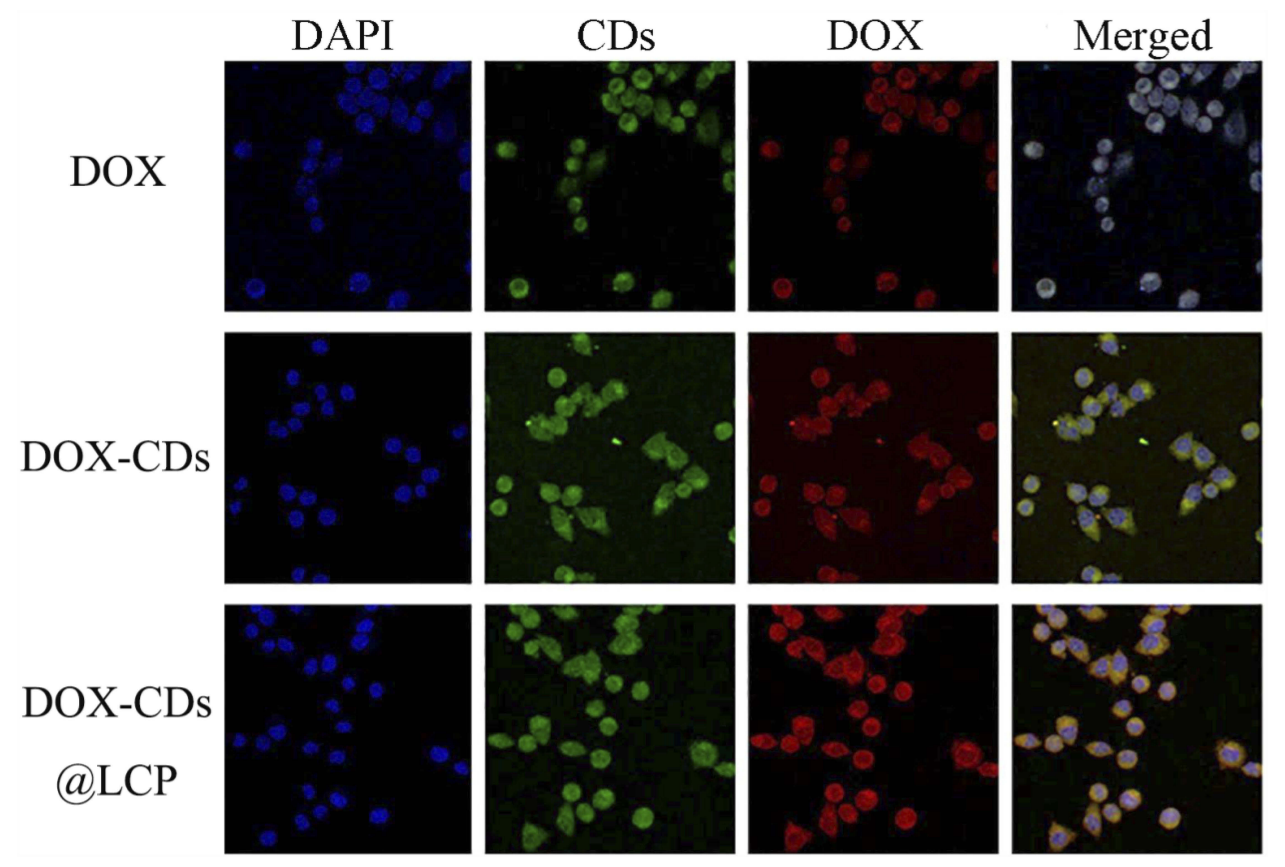

Figure 5 Confocal microscope images of HepG2 cells incubated with DOX, DOX-CDs, DOX-CDs@LCP for 4 hrs. Cell nuclei were stained with DAPI and the colocalization was confirmed by the intensity of cells by overlaying the fluorescent signals. Scale bar: $20 \mu \mathrm{m}$.

group showed a rapid increase of tumor size during the experiment, and it is interesting that the tumor size of CDs increases, whereas, DOX, DOX-CDs and DOX-CDs @LCP greatly inhibited the tumor growth.

Figure 7B describes the change of body weight of all mice. The Con and CD group showed a slight decrease during treatment and others showed a high level than Con; the significant weight loss was observed in CDs and Con groups compared to another. The free DOX displayed a slight reduction in the first 9 days, but a rapid loss after 9 days; it was likely to show systematic toxicity of DOX in whole- body biodistribution behavior in therapy. In contrast, the controlled release of DOX from DOX-CDs@LCP showed less systemic toxicity as shown by the evidence by little loss of body weight among other drug-treated groups, indicating that DOX-CDs@LCP was safe. All of mice were sacrificed and tumors were taken out after 19 days of treatment. Furthermore, Figure 7C shows the tumor weight of every group on the 19th day. We can know that the tumor size of Con and CD group was bigger than others, and the smallest was DOX-CDs@LCP group, followed by DOX-CD group and DOX group.

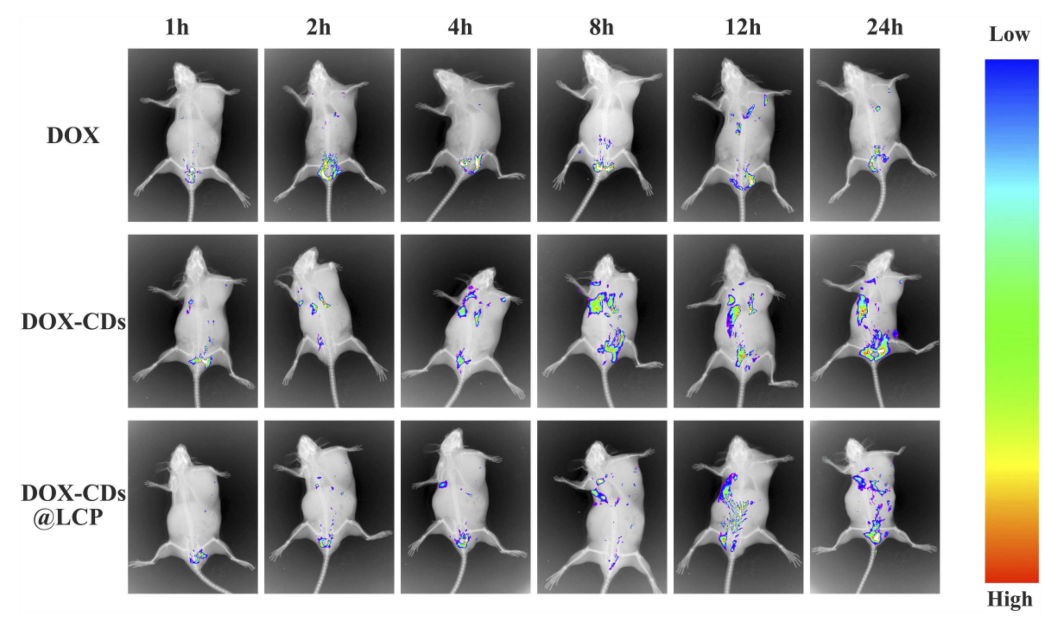

Figure 6 Real-time fluorescence imaging of tumor-bearing mice $(\mathrm{H} 22)$ after injection of DOX, DOX-CDs and DOX-CDs@LCP by tail vein. 

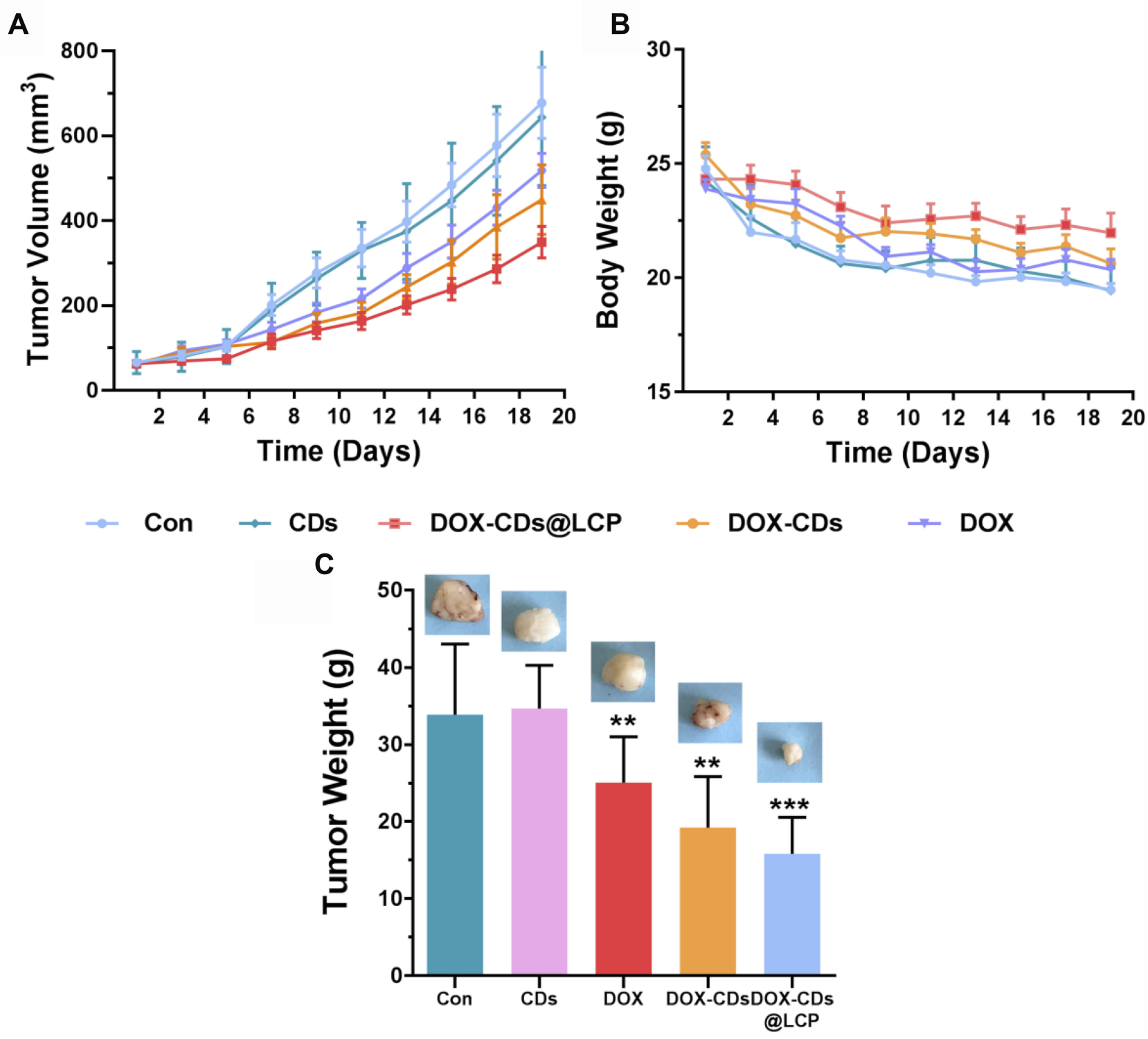

Figure 7 The mean tumor volume $(\mathbf{A})$, body weight $(\mathbf{B})$ and tumor weight $(\mathbf{C})$ of Kunming mice bearing $\mathrm{H} 22$ cells, on intravenous administration of the different formulation $(\mathrm{n}=10)$. $* * \mathrm{P}<0.01$, and $* * * \mathrm{P}<0.001$.

All these results suggest that DOX-CDs@LCP can accumulate in the tumor and stably release DOX from DOX-CDs @LCP, thereby enhancing the antitumor activity. The LCP could be regarded as an excellent delivery system. Finally, the drug in the delivery system could reduce the toxicity of full body and reach a higher therapeutic effect.

In comparison, the DOX-CDs@LCP group had the highest anti-tumor activities among the drug-treated groups,making the tumor smaller and regressed. It may be an attribution for the $\mathrm{pH}$-sensitive effect of LCP induced the drug to the tumor sites, combined functional therapy of DOX and nuclear targeted of DOX-CDs. This result was in alignment with our previous studies, and the trend is that the CDs-DOX could penetrate into the nucleus of tumor for achieving antitumor activity. Therefore, we have reasons to suppose that the higher therapeutic potential is due to the high DOX concentration accumulated in tumors.

\section{Conclusion}

In this study, we successfully synthesized a novel $\mathrm{pH}$ sensitive biodegradable DOX-CDs@LCP and delivered DOX to the tumor sites via EPR effect. The embedded CDs in LCP nanoparticles not only play an excellent role of confocal imaging contrast agent or fluorescent probe, but also enhance the loading capacity of the LCP and reduce drug premature releasing. In in vitro assays, DOX-CDs 
@ LCP showed a significant $\mathrm{pH}$-response release behavior in low pH and a slower speed release than DOX@LCP in normal $\mathrm{pH}$. More importantly, the in vivo antitumor activity study demonstrated that it had the highest antitumor capacity among all groups and the lowest systematic toxicity. These results indicated that DOX-CDs@LCP NP is a nonfouling functionalizable matrix for drug loading, imaging agent, tumor specifically accumulative and $\mathrm{pH}$-response nanocarrier to tumor tissues. In conclusion, it provides a hopeful strategy and design of nanocarriers in tumor therapy.

\section{Disclosure}

The authors report no conflicts of interest in this work.

\section{References}

1. Guan X. Cancer metastases: challenges and opportunities. Acta Pharm Sin B. 2015;5(5):S2211383515001094.

2. Jeong KH, Woo HS, Kim CJ, et al. Formulation of a modified-release pregabalin tablet using hot-melt coating with glyceryl behenate Int J Pharm. 2015;495(1):1-8.

3. Fu J, Wang H. Precision diagnosis and treatment of liver cancer in China. Cancer Lett. 2017;412:283.

4. Chamberlain C, Collin SM, Hounsome L, et al. Equity of access to treatment on the cancer drugs fund: a missed opportunity for cancer research? J Cancer Policy. 2015;5:25-30.

5. Siyuan W, Zhenzhen M, Yuanheng M, et al. Dopamine enhances the response of sunitinib in the treatment of drug-resistant breast cancer: involvement of eradicating cancer stem-like cells. Biochem Pharmacol. 2015;95(2):98-109.

6. Zhang NN, Yu RS, Xu M, et al. Visual targeted therapy of hepatic cancer using homing peptide modified calcium phosphate nanoparticles loading doxorubicin guided by $\mathrm{T} 1$ weighted MRI Nanomedicine. 2018;14(7):2167-2178.

7. Chen Q, Ding H, Zhou J, et al. Novel glycyrrhetinic acid conjugated $\mathrm{pH}$-sensitive liposomes for the delivery of doxorubicin and its antitumor activities. RSC Adv. 2016;6(22). 10.1039.C1036RA01580H

8. Shi M, Zhao X, Zhang J, et al. pH-responsive hybrid nanoparticle with enhanced dissociation characteristic for siRNA delivery. Int $J$ Nanomedicine. 2018;13:6885-6902.

9. Zhang J, Kang C, Ying D, et al. Self-assembly of $\mathrm{pH}-$-responsive dextran-g-poly(lactide-co-glycolide)-g-histidine copolymer micelles for intracellular delivery of paclitaxel and its antitumor activity. RSC Adv. 2016;6(28). 10.1039.C1035RA22463B

10. Huang XQ, Yang HY, Luo T, et al. Hollow mesoporous zirconia delivery system for biomineralization precursors. Acta Biomater 2018;67:366-377.

11. Gao X, Zhai M, Guan W, et al. Controllable synthesis of a smart multifunctional nanoscale metal-organic framework for magnetic resonance/optical imaging and targeted drug delivery. ACS Appl Mater Interfaces. 2017;9(4):3455-3462.

12. Hou X, Zeng F, Du F, et al. Carbon-dot-based fluorescent turn-on sensor for selectively detecting sulfide anions in totally aqueous media and imaging inside live cells. Nanotechnology. 2013;24 (33):335502.

13. Demir B, Lemberger MM, Panagiotopoulou M, et al. Tracking hyaluronan: molecularly imprinted polymer coated carbon dots for cancer cell targeting and imaging. ACS Appl Mater Interfaces. 2018;10 (4):3305-3313.
14. Xue X, Fang T, Yin L, et al. Multistage delivery of CDs-DOX/ICGloaded liposome for highly penetration and effective chemo-photothermal combination therapy. Drug Deliv. 2018;25(1):1826-1839.

15. Yang L, Jiang W, Qiu L, et al. One pot synthesis of highly luminescent polyethylene glycol anchored carbon dots functionalized with a nuclear localization signal peptide for cell nucleus imaging. Nanoscale. 2015;7(14):6104-6113.

16. Yuan Y, Guo B, Hao L, et al. Doxorubicin-loaded environmentally friendly carbon dots as a novel drug delivery system for nucleus targeted cancer therapy. Colloids Surf B Biointerfaces. 2017;159:349-359.

17. Yilmaz S, Atessahin A, Sahna E, et al. Protective effect of lycopene on adriamycin-induced cardiotoxicity and nephrotoxicity. Toxicology. 2006;218(2):164-171.

18. Octavia Y, Tocchetti CG, Gabrielson KL, et al. Doxorubicin-induced cardiomyopathy: from molecular mechanisms to therapeutic strategies. J Mol Cell Cardiol. 2012;52(6):1213-1225.

19. Abo-Salem OM. The protective effect of aminoguanidine on doxorubicin-induced nephropathy in rats. $J$ Biochem Mol Toxicol. 2012;26(1):1-9.

20. Zhang J, Zhao X, Chen Q, et al. Systematic evaluation multifunctional paclitaxel-loaded polymer -ic mixed micelles as a potential anticancer remedy to overcome multidrug resistance. Acta Biomater. 2017;50:381-395.

21. Olton D, Li J, Wilson ME, et al. Nanostructured calcium phosphates (NanoCaPs) for non-viral gene delivery: influence of the synthesis parameters on transfection efficiency. Biomaterials. 2007;28 (6):1267-1279.

22. Bobo D, Robinson KJ, Islam J, et al. Nanoparticle-based medicines: a review of FDA-approved materials and clinical trials to date. Pharm Res. 2016;33(10):2373-2387.

23. Habraken W, Habibovic P, Epple M, et al. Calcium phosphates in biomedical applications: materials for the future? Mater Today. 2016;19(2):S136970211500317X.

24. Zhang Y, Bush X, Yan B, et al. Gemcitabine nanoparticles promote antitumor immunity against melanoma. Biomaterials. 2019;189:48-59.

25. Sokolova V, Knuschke T, Kovtun A, et al. The use of calcium phosphate nanoparticles encapsulating toll-like receptor ligands and the antigen hemagglutinin to induce dendritic cell maturation and T cell activation. Biomaterials. 2010;31(21):5627-5633.

26. Li J, Chen YC, Tseng YC, et al. Biodegradable calcium phosphate nanoparticle with lipid coating for systemic siRNA delivery. J Control Release. 2010;142(3):416-421.

27. Ruan S, Cao X, Cun X, et al. Matrix metalloproteinase-sensitive size-shrinkable nanoparticles for deep tumor penetration and $\mathrm{pH}$ triggered doxorubicin release. Biomaterials. 2015;60:100-110.

28. Ruan S, He Q, Gao H. Matrix metalloproteinase triggered size-shrinkable gelatin-gold fabricated nanoparticles for tumor microenvironment sensitive penetration and diagnosis of glioma. Nanoscale. 2015;7(21):9487-9496.

29. Tong R, Hemmati HD, Langer R, et al. Photoswitchable nanoparticles for triggered tissue penetration and drug delivery. $J$ Am Chem Soc. 2012;134(21):8848-8855.

30. Cliff W, Triantafyllos S, Jian C, et al. Multistage nanoparticle delivery system for deep penetration into tumor tissue. Proc Natl Acad Sci U S A. 2011;108(6):2426-2431.

31. Poole RL, Rupp CA. Calcium and phosphorus in neonatal parenteral nutrition solution. J Parenter Enteral Nutr. 1983;7(4):358-360.

32. Ru D, Ding C, Liu L, et al. pH sensitive triptolide-loaded liposome calcium phosphate nanoparticles exhibit enhanced anti-tumor activities against ovarian cancer without damaging the reproductive system. J Biomed Nanotechnol. 2017;13(11):1413-1424.

33. Zeng Y-W, Ma D-K, Wang W, et al. N, S co-doped carbon dots with orange luminescence synthesized through polymerization and carbonization reaction of amino acids. Appl Surf Sci. 2015;342:136-143. 
34. Shu Y, Lu J, Mao QX, et al. Ionic liquid mediated organophilic carbon dots for drug delivery and bioimaging. Carbon. 2017;114:324-333.

35. Ruizheng L, Min W, Evans DG, et al. Inorganic nanomaterials for bioimaging, targeted drug delivery and therapeutics. Chem Commun (Camb). 2014;50(91):14071-14081.
36. Ya-Ping Sun BZ, Lin Y, Wei Wang KA, et al. Quantum-sized carbon dots for bright and colorful photoluminescence. J Rehabil Res Dev. 2013;50(9):vii-viii.

\section{Publish your work in this journal}

The International Journal of Nanomedicine is an international, peerreviewed journal focusing on the application of nanotechnology in diagnostics, therapeutics, and drug delivery systems throughout the biomedical field. This journal is indexed on PubMed Central, MedLine, CAS, SciSearch ${ }^{\mathbb{R}}$, Current Contents ${ }^{\mathbb{R}} /$ Clinical Medicine, $^{2}$
Journal Citation Reports/Science Edition, EMBase, Scopus and the Elsevier Bibliographic databases. The manuscript management system is completely online and includes a very quick and fair peer-review system, which is all easy to use. Visit http://www.dovepress.com/ testimonials.php to read real quotes from published authors. 